\title{
Evaluación del uso de larvas de Mosca doméstica (Musca domestica L.) como tratamiento biológico de residuos orgánicos municipales
}

\section{Evaluation of the use of house fly larvae (Musca domestic L.) as a biological treatment of municipal organic wastes}

\author{
Reynaldo Salas Sucaticona ${ }^{l}$, Yuly Mercedes Apaza Gómez ${ }^{l}$ Ebed David Paredes Rodríguez ${ }^{l}$ \\ ${ }^{l}$ Escuela Profesional de Ingeniería Ambiental y Forestal, Universidad Nacional de Juliaca, Perú.
}

\begin{abstract}
Resumen Se determinó la eficacia del tratamiento biológico de residuos orgánicos municipales usando larvas de mosca doméstica (Musca domestica L.). Se colectaron aleatoriamente $48 \mathrm{~kg}$ de residuos orgánicos, divididos en 9 unidades experimentales, distribuidas en grupos de 3 unidades; cada uno sometido a tres (3) tratamientos, el T1: residuos de mercado (RM), T2: residuos de restaurante (RR) y T3: residuos domiciliarios (RD); consecutivamente se estimó el índice de reducción y tasas de crecimiento. Por medio del Software SPSS versión 25.0 y Minitab versión 18.1.0 se aplicó un Análisis de varianza (ANOVA), con un diseño completo al azar (DCA). Se reportó índices de reducción (WRI) significativamente diferentes en todos los tratamientos ( $<<0.05)$, que son los residuos domiciliarios (RD) los que presentaron un mayor índice de reducción de 1.167\%/día. Con respecto a la eficiencia de conversión de alimentos ingeridos (ECI), no se observaron entre tratamientos diferencias significativas $(\mathrm{p}<0.05)$. No obstante, con respecto al incremento de longitud y peso de larvas, se observaron diferencias significativas $(\mathrm{p}<0.05)$ entre las tasas de crecimiento absoluta (TAC), relativa (TRC) y especifica (TEC) que son en peso y talla significativamente diferentes en todos los tratamientos, con un máximo aumento en residuos domiciliarios (RD) con $0.333 \mathrm{~mm} /$ día y $2.254 \mathrm{mg} /$ día respectivamente. Finalmente, se demostró que las larvas de mosca doméstica son eficaces en la degradación de residuos orgánicos, observándose que, a mayor grado de reducción de residuos orgánicos, mayores son las tasas de crecimiento de las larvas (TAC), (TRC) y (TEC), y mayor es el índice de reducción de residuos.
\end{abstract}

Palabras clave Índice de reducción, larva, Musca domestica L., residuo orgánico municipal y tratamiento biológico.

\begin{abstract}
The efficacy of the biological treatment of municipal organic wastes using house fly larvae (Musca domestica L.) was determined. $48 \mathrm{~kg}$ of organic waste were randomly collected, divided into 9 experimental units, distributed in groups of 3 units; each one subjected to three (3) treatments, T1: market waste (MR), T2: restaurant waste (RR) and T3: household waste (RD); consecutively, the reduction index and growth rates were estimated. Using the SPSS Software version 25.0 and Minitab version 18.1.0, an analysis of variance (ANOVA) was applied, with a complete randomized design (CRD). Reduction rates (WRI) were reported significantly different in all treatments $(\mathrm{p}<0.05)$, being household waste (RD) showing the highest rate of reduction of $1.167 \% /$ day. However, regarding the ingested food conversion efficiency (ECI) no significant differences were observed among treatments $(\mathrm{p}<0.05)$. On the other hand, with respect to the increase in length and weight of larvae, significant differences $(\mathrm{p}<0.05)$ were observed between the absolute (TAC), relative (TRC) and specific (TEC) growth rates, being significantly different in weight and length in all treatments, with a maximum increase in household waste (RD) with $0.333 \mathrm{~mm} /$ day and $2.254 \mathrm{mg} /$ day respectively. Finally, it was demonstrated that house fly larvae are effective in the degradation of organic waste, observing that the higher the reduction of organic waste, the higher the growth rates of the larvae (TAC), (TRC) and (TEC), and the higher the rate of waste reduction.
\end{abstract}

Keywords Reduction rate, larvae, Musca domestica L., municipal organic waste, biological treatment

* Corresponding author: reynaldosalassucaticona@gmail.com

\section{Introducción}

Según la Organización de las Naciones Unidas para la Agricultura y la Alimentación (FAO), los residuos de alimentos incluyen cualquier sustancia sana o comestible que se desperdicia, se pierde o se degrada en cada etapa de la cadena de suministro de alimentos, donde se observa que 
anualmente se pierden entre 1.300 y 1.600 millones de toneladas de alimentos, como verduras frescas, frutas $\mathrm{y}$ productos cárnicos, de panadería y lácteos, lo que supone un tercio de los alimentos producidos mundialmente para el consumo humano, afectando varios recursos naturales. Los residuos sólidos y su gestión a nivel mundial son considerados uno de los problemas más peligrosos y difíciles de manejar, por lo que representan uno de los más graves e inmediatos problemas ambientales que deben enfrentar los países del tercer mundo [1].

Según informe presentado por el Banco Mundial, se estima que a nivel mundial se generan actualmente 1.3 mil millones de toneladas de residuo, con proyecciones para el 2025 de un incremento por año a 2.2 mil millones de toneladas. De estos, alrededor del $19 \%$ se somete a la recuperación de materiales mediante el reciclaje, el $4 \%$ por medio del compostaje y un $11 \%$ se trata mediante la incineración con medios modernos. La eliminación de los desechos sólidos en terrenos abiertos o en vertederos diseñados incorrectamente puede generar efectos adversos al medio ambiente y a la salud humana. En marco de la búsqueda de solución a tantos problemas ambientales y de salud generados por la mala disposición y manejo de este tipo de desecho mundialmente, se han establecido normas para la reutilización de residuos orgánicos diferenciados de los residuos municipales con fines agrícolas, donde es el compostaje uno de los métodos biológicos adecuados y más conocidos para transformar los desechos orgánicos en enmiendas útiles para el suelo [2].

En el caso específico de la ciudad de Juliaca, son recogidos 169.30 ton/día de residuos con una generación per cápita de $0.71 \mathrm{~kg} / \mathrm{hab} /$ día es el $40 \%$ residuos orgánicos de origen domiciliario y de mercados de abasto, residuos que son dispuestos en un botadero transitorio, que se convierten en un problema ambiental debido a la presencia de procesos biológicos de descomposición que originan agentes contaminantes, como lixiviados y biogases contaminantes de suelos, agua y aire [3].

Las prácticas de generación, transporte y eliminación de residuos son las principales fuentes de emisiones de gases de efecto invernadero (GEI) para las actividades de gestión de residuos. Estos son la mayor generación de lixiviados y gases de efecto invernadero, asociados a problemas altamente técnicos que actualmente no han sido resueltos. Esta situación ha producido una continua acumulación de residuos, generando niveles críticos en casi todas las regiones del mundo, que convierten a los residuos orgánicos como uno de los problemas ambientales que necesitan ser manipulados de modo sostenible y así minimizar el riesgo para la salud humana y disminuir las cargas ambientales [4].

Por consiguiente, con la intención de responder a la mencionada problemática ambiental, son muchas las investigaciones que han planteado ciertas alternativas enfocadas en el abordaje de los problemas generados por el tratamiento de residuos orgánicos [5], los hallazgos demostraron que los residuos orgánicos cuentan con un alto potencial para ser valorizados a través de tratamientos biológicos, dado que los residuos orgánicos pueden transformarse en productos finales, como enmiendas agrícolas, energía y proteínas, que consideran a las larvas de insectos como el tratamiento biológico o de bioconversión que presentan una mayor efectividad, es capaces de digerir residuos orgánicos con una reducción de su volumen en un 50-80\% [6].

El propósito de esta investigación fue realizar un estudio basado en el desarrollo de nuevas técnicas direccionadas al manejo de residuos sólidos orgánicos, generando beneficios ambientales y económicos a través de la propuesta de métodos alternativos con beneficios ambientales, mostrando a las larvas de mosca doméstica ( $M$. domestica L.) como un tratamiento de residuos ideal para ser criadas y cosechadas de manera simple y económica para contribuir significativamente a la reducción del potencial contaminante del residuo.

El objetivo de esta indagación fue determinar la eficacia del tratamiento biológico de residuos orgánicos municipales usando larvas de mosca doméstica (Musca domestica L.) en la ciudad de Juliaca, Perú.

\section{Antecedentes}

La "bioconversión", basada en los insectos, representa un método económicamente viable, el cual tiene la capacidad de convertir grandes cantidades de residuos orgánicos de origen alimenticio en materia prima de gran valor ambiental y económico, como la elaboración de piensos como alimentos de animales, el cual presenta una elevada biomasa de insectos, alimentos para humanos, elaboración de compuestos industriales secundarios como biocombustibles, lubricantes, productos farmacéuticos, entre otros, así como el uso de los residuos alimentarios sobrantes como materia orgánica con grandes cantidades de nutrientes [7].

Una de las especies de insectos en la que más se ha estudiado la capacidad de bioconversión ha sido la mosca soldado negro Hermetia illucens L. Con el propósito de analizar el potencial de las larvas de la mosca de soldado negra (MSN) en el proceso de bioconversión de residuos orgánicos municipales, analizar la calidad del compost producido y el contenido nutricional de larvas de MSN (gusanos) se experimentó con larvas de unas 2 a 3 semanas, obteniendo como productos finales de la bioconversión larvas con elevado contenido nutritivo y compost. Se pudo comprobar que las larvas podían reducir los residuos orgánicos (residuos orgánicos municipales) en un $47.75 \%$, presentando estas una capacidad para consumir $26.1508 \mathrm{~g}$ de residuos / g de gusano, observando en estos un contenido de proteína, grasa bruta y cenizas del $41.8 \%, 14.63 \%$ y $9.12 \%$, respectivamente [8]. 
De igual manera ha sido aplicada en la bioconversión de residuos orgánicos de la industria del café, estudiando su eficiencia de conversión de alimentos ingeridos (ECI) y el índice de reducción de residuos (WRI, por sus siglas en inglés), reportando que las larvas que presentan una tasa de alimentación de $200 \mathrm{mg} / \mathrm{larva}$ /día se desarrollan en un tiempo más corto, demostrando que este insecto, a pesar de tener una lenta tasa de crecimiento, tiene la capacidad de convertir los restos de café molido [9].

Con la intención de un uso eficiente y factible de los desechos de alimentos y de generar beneficios económicos y ambientales, se utilizó la larva de $M$. domestica L. como agente bioconversor para obtener la proteína de gusano con un valor añadido, aceite y fertilizantes orgánicos. Se criaron moscas adultas, con el fin de cultivar larvas (gusanos) y valorizar los residuos. A partir de los resultados experimentales se demostró que de cada $1.000 \mathrm{~g}$ de medio de cultivo compuesto de $700 \mathrm{~g}$ de residuos de alimentos y $300 \mathrm{~g}$ de adyuvante se podrían aprovechar 1,5 g de huevos de $M$. domestica $\mathrm{L}$. en condiciones de cultivo adecuadas en tan solo 4 días, con una reducción de alimentos en un $42.95 \pm 0.25 \%$, utilizando los restos del medio de cultivo como buenos fertilizantes orgánicos.La obtención de $53.08 \mathrm{~g}$ de gusanos secos con $57.06 \pm 2.19 \%$ de proteínas y $15.07 \pm 2.03 \%$ de aceite, proteínas que han sido destacadas por sus actividades farmacológicas, que son una buena materia prima en el campo de la medicina y la alimentación animal. Por otro lado, el aceite de gusano representa una potencial materia prima alternativa para la producción de biodiésel, que encuentra en este estudio un $87.71 \%$ de biodiésel [10].

\section{Marco teórico}

\subsection{Residuos sólidos y clasificación}

Según la procedencia del residuo, se clasifican como residuos urbanos; residuos industriales; residuos creados en los servicios; y residuos provenientes de construcciones, entre los urbanos se encuentran los desechos orgánicos, el vidrio, el papel entre otros [11].

\subsection{Características de los residuos sólidos orgánicos}

Todo aquel residuo originado biológicamente, es aquel que presenta tendencia a descomponerse por la acción de organismos vivos, los cuales generan gases como el dióxido de carbono, metano, entre otros; el de lixiviados en los lugares donde finalmente se sitúan, recurso que bien tratado puede revalorizarse y mejorar los suelos a través de la producción de abono, vermicompost, compost entre otros [12].

\subsection{Tratamientos físicos}

Los residuos generalmente, pueden separarse a través de ciertas técnicas mecánicas y/o físicas, separándolos en fracciones para posteriormente ser sometidos a adicionales tratamientos como digestión anaeróbica, reciclaje, biogas, combustión entre otros [13].

\subsection{Tratamientos Biológicos 3.4.1Bioconversión}

Es un proceso muy productivo, donde se utiliza agentes biológicos de rápida aparición, el cual es muy aplicado en tecnología de alimentos, en estudios ambientales y de salud. Es considerado un proceso de transformación de restos alimentarios y algunos subproductos. Con el fin de generar instrumentos y métodos enfocados según el interés del investigador, que se ha logrado a través del tiempo la transformación de desechos orgánicos en recursos beneficiosos para el ambiente y la economía. En el caso de la comercialización de la bioconversión basada en insectos, esta representa un cambio prometedor a la hora de ofrecer opciones alternativas para la reducción de los residuos alimentarios [14].

\subsubsection{Vermicompostaje}

Este tratamiento consiste en la transformación rápida y efectiva de la materia orgánica en abono por medio del uso de métodos limpios y sostenibles con la intención de producir residuos aprovechables. Es apreciado como una potencial opción para la bio-oxidación, degradación y estabilización de residuos orgánicos. Para llevar a cabo este tratamiento se usan lombrices para transformar los restos orgánicos en un material análogo al humus, pero en este caso conocido como vermicompost [15].

3.5 Características de la Mosca doméstica $M$. domestica $\mathbf{L}$.

La especie $M$. domestica L. es un insecto asociado comúnmente a los humanos, y a los animales de corral, por lo que son consideradas sinantrópicas. Estas generalmente transportan en asociación ciertos organismos que generan enfermedades, que se pueden reproducir en materia vegetal en descomposición o excreciones de animales, por lo que puede adquirir agentes patógenos [16].

\subsubsection{Ciclo vital de la Mosca domética $M$. domestica $\mathbf{L}$.}

Su ciclo de vida está conformado por 4 estadios conocidos como huevo, larva, pupa y adulto. Este insecto exhibe una metamorfosis completa, en el caso de la larva que posee tres fases larvarias, donde la última es la de mayor tamaño con una talla máxima de 5-13 mm [17].

\subsection{Variables para analizar el consumo de alimentos}

Entre las variables usadas para comprobar el consumo de alimentos se encuentran el índice de reducción de residuos (WRI) y el índice de eficiencia de conversión de alimentos ingeridos (ECI).

\subsection{1 Índice de reducción de residuos}

Es primordial en la evaluación de la cantidad de alimento reducido a través del uso del índice de reducción de residuos. El tiempo en que se degradará un alimento va a variar de acuerdo con la cantidad de alimento a degradar, por lo que, se calcula a través de la siguiente ecuación: 
$W R I=D / t x 100$, Dónde $D=(W-R / W) / t$

Donde: $\mathrm{D}=$ degradación global. $\mathrm{t}=$ número total de días en que las larvas se alimentaron. $\mathrm{R}=$ residuo después del tiempo $\mathrm{t}$.

\subsubsection{Eficiencia de conversión de los alimentos ingeridos (ECI)}

Índice también conocido como "Eficiencia de crecimiento", viene representado por una escala de la cantidad de comida aproximada que ingiere un animal y se convierte en crecimiento a través del aumento de biomasa (talla y peso), se cálcula a través de la siguiente ecuación:

$$
E C I=(\text { Ganancia de peso } / \text { peso del alimento ingerido }) \times 100
$$

\subsection{Variables para examinar la tasa de crecimiento}

Esta tasa de crecimiento se puede determinar a través de la medición de las siguientes variables propuestas por Wootton (1991):

\subsubsection{Tasa específica de crecimiento (TEC) \\ $T E C=100[\ln (W F)-\ln (W I)] / t$}

Donde: $\ln (\mathrm{WF})=$ logaritmo natural del peso húmedo final $\ln$. $(\mathrm{WI})=\operatorname{logaritmo}$ natural del peso húmedo inicial. $\mathrm{t}=$ tiempo (días). Los resultados se representan en \%. día-1.

\subsubsection{Tasa absoluta de crecimiento (TAC)}

$$
T A C=(Y 2-Y 1) /(T 2-T 1)
$$

Donde: $\mathrm{Y} 1$ y $\mathrm{Y} 2=$ Peso o Talla al inicio y al final del experimento. T1 Y T2= Tiempo inicial y final. Los resultados se representan en $\mathrm{cm}$ y $\mathrm{mg}$.

\subsubsection{Tasa relativa de crecimiento (TRC)}

$$
T R C=(Y 2-Y 1) / Y 1(T 2-T 1) \mathrm{X} 100
$$

Donde: $\mathrm{Y} 1$ y $\mathrm{Y} 2=$ Peso o Talla inicial y final. $\mathrm{T} 1 \mathrm{Y} \mathrm{T} 2=$ Tiempo inicial y final. Los resultados se representan $\mathrm{cm}$ y $\mathrm{mg}$.

\section{Materiales y métodos}

El presente estudio científico siguió una orientación cuantitativa, dentro de la modalidad de diseño experimental totalmente aleatorio (DCA), esto con la finalidad de verificar si existían significativas diferencias entre los tratamientos aplicados. Se manejó la variable residuos orgánicos municipales como independiente y ver como era su influencia sobre variable dependiente.

La investigación se realizó bajo un diseño experimental [18] conformado por grupos aleatorizados y post prueba, por lo que se aplicó el siguiente diseño experimental:

$$
\begin{aligned}
& R G 1 \rightarrow X \rightarrow \mathrm{O} 1 \\
& R G 2 \rightarrow X \rightarrow \mathrm{O} 2 \\
& R G 3 \rightarrow X \rightarrow \mathrm{O} 3
\end{aligned}
$$

Donde: R: Asignación de residuos orgánicos al azar.
G1: Grupo 1, conformado por residuos orgánicos sin tratamiento, provenientes del mercado.

G2: Grupo 2, conformado por residuos orgánicos sin tratamiento, provenientes de restaurantes.

G3: Grupo 3, conformado por residuos orgánicos sin tratamiento, provenientes de diferentes domicilios.

X: Tratamiento de bioconversión con Mosca doméstica ( $M$. domestica L.).

O1: Valor del índice de reducción, tasas de crecimiento y contenido proteico posterior al tratamiento en el grupo 1.

O2: Valor del índice de reducción, tasas de crecimiento y contenido proteico posterior al tratamiento en el grupo 2 .

O3: Valor del índice de reducción, tasas de crecimiento y contenido proteico posterior al tratamiento en el grupo 3.

Antes del análisis de los datos obtenidos se procedió a verificar la normalidad a través de la prueba de Shapiro-Wilk (Herrera \& Fontalvo, 2011). De igual manera, se aplicó la prueba de homogeneidad de varianza de Levene, con el propósito de verificar la variabilidad entre diferentes grupos con un nivel de significancia de (0.05).

Las muestras de residuos orgánicos fueron obtenidas de los mercados de abasto más importantes de la ciudad de Juliaca, los cuales se ubican en el Altiplano Peruano, distrito de Juliaca, Departamento de Puno, Perú a $3826 \mathrm{msnm}$ en las coordenadas 376645.3 de latitud Sur y 8287146 de longitud Oeste. Se realizaron visitas previas para la identificación de los lugares de muestreo de residuos orgánicos más adecuados, ver tabla 1.

Tabla 1. Principales mercados de abasto muestreados en Juliaca y su ubicación

\begin{tabular}{|l|l|c|c|}
\hline \multicolumn{4}{|c|}{ Coordenadas UTM } \\
\hline $\begin{array}{c}\text { Mercados de } \\
\text { abasto }\end{array}$ & Zona & Este & Norte \\
\hline Santa Bárbara & $19 \mathrm{~L}$ & 378040.27 & 8286998.39 \\
\hline Túpac Amaru & $19 \mathrm{~L}$ & 379202.44 & 8287217.14 \\
\hline Las Mercedes & $19 \mathrm{~L}$ & 378009.57 & 8287723.06 \\
\hline Cerro Colorado & $19 \mathrm{~L}$ & 379090.72 & 8285828.45 \\
\hline
\end{tabular}

Fuente: Elaboración propia.(GPS Garmin Map 64s).

\subsection{Materiales y equipos}

Al realizar esta investigación se necesitó una serie de equipos y materiales entre los que se puede nombrar:

- Envases de polietileno de alta densidad $(20 \times 20 \mathrm{~cm})$.

- Pinzas, crisoles, luna reloj, morteros de porcelana, tamiz, envases de vidrio (Boro 3.3.5)

- Termo-hidrómetro Digital (Boeco HTC-1)

- GPS de mano (Garmin Map 64s)

- Potenciómetro (Hanna HI 98130)

- Balanza analítica (Kern modelo ABS 220-4N)

- Estufa (Selecta Modelo Conterm 2000200)

- Mufla (Select-Horn modelo TFT) 
Salas (et al): Evaluación del uso de larvas de Mosca doméstica (Musca domestica L.) como tratamiento biológico de residuos orgánicos municipales

- Microscopio electrónico (DM-500/Cámara Leica modelo ICC50-W)

- Vernier digital (Tool Size modelo Premium 0 - 120 mm).

\subsection{Reactivos}

- Agua purificada (QP)

- Etanol $\left(\mathrm{C}_{2} \mathrm{H}_{5} \mathrm{OH}\right.$ al $\left.96 \%\right)$

- Hipoclorito de sodio ( $\mathrm{NaClO}$ al $10 \%)$

- Solución buffer pH 4.7 y 10 (reactivos manipulados en un grado analítico).

\subsection{Población y muestra \\ 4.3.1 Población}

Conformada por residuos orgánicos de tres (3) orígenes: de mercado, restaurantes y domiciliarios, estos provenientes de los mercados de abasto principales de la ciudad de Juliaca (Santa Bárbara, Túpac Amaru, Las Mercedes y Cerro Colorado). Se afirma que el $77.15 \%$ de estos residuos son orgánicos, el $18.45 \%$ inorgánicos y solo el $4.40 \%$ mixto (residuos sanitarios, pañales, papel sanitario, entre otros.). Tomando en cuenta para este estudio la fracción orgánica compuesta por restos de alimentos, huesos, follaje y restos de cartón.

\subsubsection{Muestra}

Aleatoriamente se tomaron muestras de residuos orgánicos de $48 \mathrm{~kg}$ (Casal \& Mateu, 1992), conformadas por $16 \mathrm{~kg}$ para cada tipo de residuo orgánico (provenientes de mercado, restaurante y domiciliarios) ver tabla 2. Para el proceso de experimentación, se obtuvo una muestra representativa de $1 \mathrm{~kg}$ por tipo de residuo, aplicando el método de cuarteo considerando la guía metodológica para el desarrollo de ECRSM, MINAM, 2015.

Tabla 2. Muestra de residuos orgánicos municipales

\begin{tabular}{|c|c|c|c|c|}
\hline 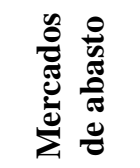 & 窇 & 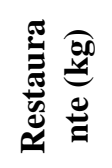 & 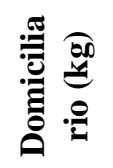 & 氶 \\
\hline $\begin{array}{c}\text { Santa } \\
\text { Bárbara }\end{array}$ & 4.02 & 4.02 & 4.01 & 12.05 \\
\hline $\begin{array}{l}\text { Túpac } \\
\text { Amaru }\end{array}$ & 4.01 & 4.03 & 4.03 & 12.07 \\
\hline $\begin{array}{c}\text { Las } \\
\text { Mercedes }\end{array}$ & 4.03 & 4.01 & 4.02 & 12.06 \\
\hline $\begin{array}{c}\text { Cerro } \\
\text { Colorado }\end{array}$ & 4.02 & 4.02 & 4.01 & 12.05 \\
\hline Total & & & & 488.23 \\
\hline
\end{tabular}

Fuente: Elaboración propia.

\subsubsection{Obtención de la información}

En consecuencia, para obtener los resultados se siguió el siguiente esquema, ver figura 1 .

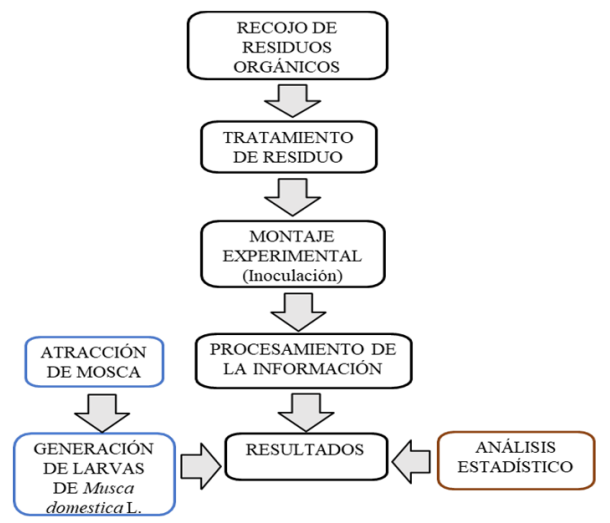

Figura 1. Vista general de la metodología aplicada. Fuente: Modificado de guía metodológica para el desarrollo de EC-RSM, MINAM, 2015.

\subsection{Determinación del índice de degradación de residuos sólidos}

Se siguió el diagrama de flujo realizado en el laboratorio de Biología de la Escuela Profesional de Ingeniería Ambiental y Forestal (EPIAF), ver figura 2. Para determinar este índice se aplicó la ecuación (1)

Degradación total de sólidos:

$$
\mathrm{D}=(\mathrm{W}-\mathrm{R}) / \mathrm{W}
$$

Donde: $\mathrm{W}=$ cantidad total de alimento suministrado. $\mathrm{R}=$

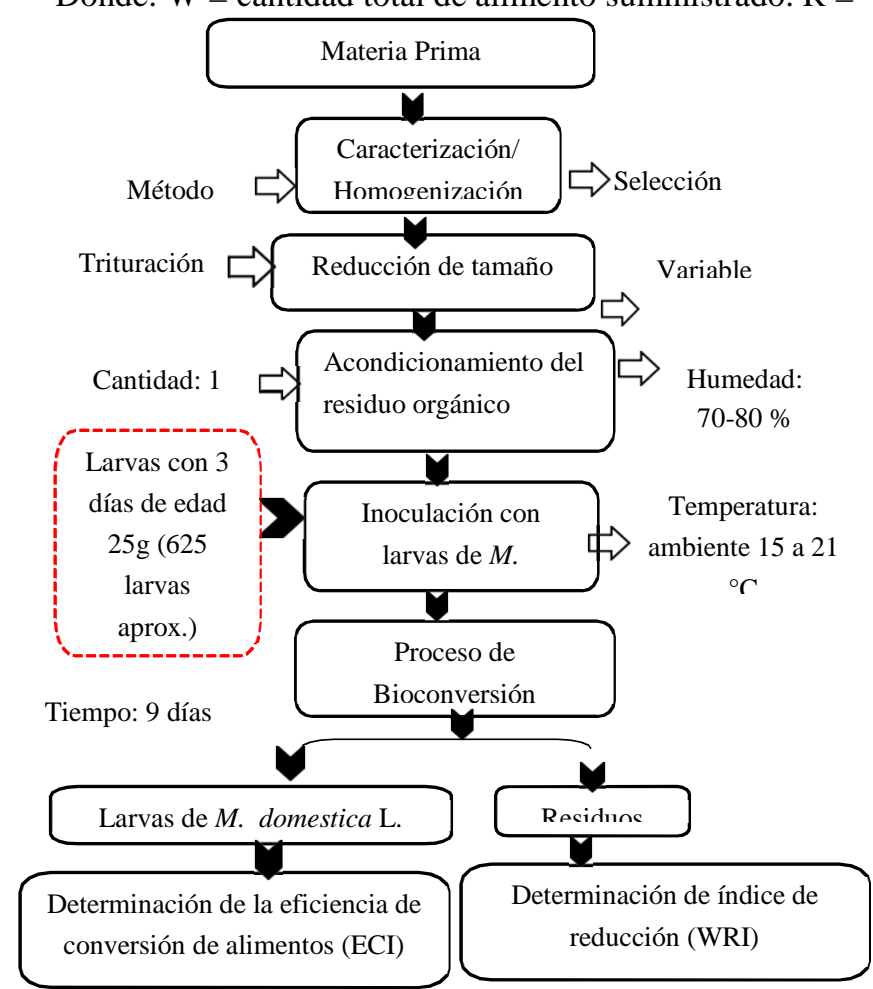

Sustrato restante

Figura 2. Diagrama de flujo para los análisis de laboratorio 


\subsection{Condiciones de laboratorio}

Al iniciar la investigación, se usó 9 bandejas de plástico con tapa y capacidad de $6 \mathrm{~kg}$. A cada tapa se le realizaron agujeros para permitir circulación de aire y se colocó tela mosquitera entre las tapas y los recipientes, con el propósito de controlar otra intrusión de insectos. Para cada tipo de residuo se triplicó el tratamiento basado en metodología de Diener et al., 2009, ver figura 3.

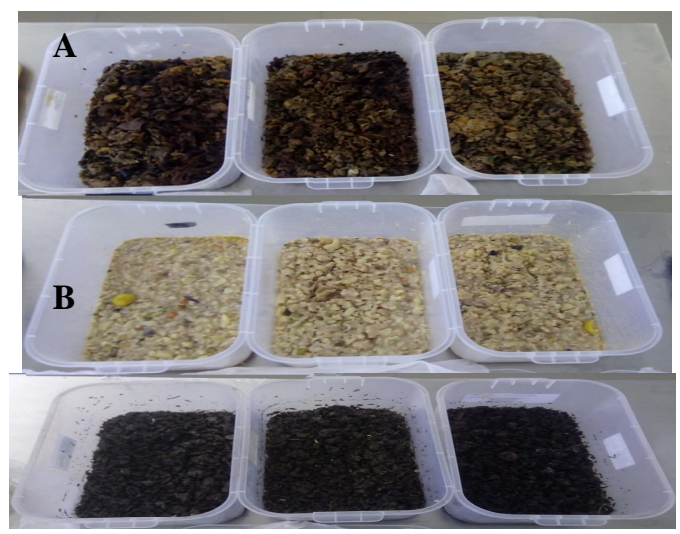

Figura 3. Acondicionamiento de residuos orgánicos: A de mercado. B de restaurante $\mathbf{C}$ domiciliario para ser inoculados con larvas de $M$. domestica $\mathrm{L}$

Fuente: Elaboración propia.

El laboratorio contó con un aislador térmico (Tecnopor) para mantener la temperatura a $20^{\circ} \mathrm{C}$ y generar un crecimiento y desarrollo óptimo de las larvas de mosca; la investigación se realizó bajo las condiciones ambientales reflejadas en la tabla 3.

Tabla 3. Condiciones experimentales en laboratorio

\begin{tabular}{|c|c|c|c|c|c|c|c|}
\hline & & & & & ñana & & arde \\
\hline 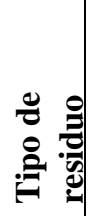 & 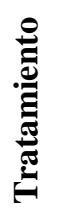 & 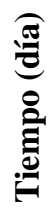 & 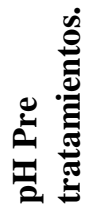 & U & 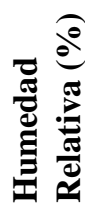 & $\bigcup_{0}$ & 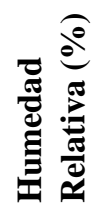 \\
\hline & T1 & 9 & 5.4 & 15 & & 21 & \\
\hline$\Xi 0$ & T1 & 9 & 5.2 & $\mathrm{a}$ & 35 & a & 27 \\
\hline$\Sigma \sigma$ & T1 & 9 & 5.3 & 21 & & 25 & \\
\hline & T2 & 9 & 6.0 & 15 & & 21 & \\
\hline है & T2 & 9 & 6.2 & $\mathrm{a}$ & 35 & a & 27 \\
\hline$\simeq \exists$ & T2 & 9 & 6.1 & 21 & & 25 & \\
\hline & T3 & 9 & 6.3 & 15 & & 21 & \\
\hline$\cdot \stackrel{\mathscr{J}}{\mathrm{B}} \mathrm{O}$ & T3 & 9 & 6.1 & $\mathrm{a}$ & 35 & $\mathrm{a}$ & 27 \\
\hline 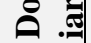 & T3 & 9 & 6.0 & 21 & & 25 & \\
\hline
\end{tabular}

Fuente: Elaboración propia.

\subsection{Atracción de larva}

Determinar el atrayente más efectivo, se aplicó una matriz de selección, donde se consideró como parámetros el costo, sanidad y capacidad de atracción; por lo que se comparó a la cabeza de res con la carne de res y la carne de pollo, determinando a la cabeza de res como el atrayente más idóneo y de bajo costo para atraer y generar larvas de mosca doméstica (M. doméstica L.).

\subsection{Estimación del índice de reducción de residuos sólidos}

$\mathrm{Al}$ estimar este índice se evaluó diariamente el peso de las bandejas, ver figura 2. Posteriormente se estimó el índice de reducción de residuos (WRI) (1) (2), y se evaluó la eficacia en la conversión de alimentos ingeridos (ECI) (3) basados en Scriber \& Slansky, 1981, calculado a través de las ecuaciones 1 y 2 respectivamente.

Índice de reducción de residuos (\%. día):

$$
\mathrm{WRI}=\mathrm{D} / \mathrm{t} \times 100
$$

Dónde: $\mathrm{D}=$ degradación total. $\mathrm{t}=$ tiempo en días

Eficiencia de conversión de los alimentos ingeridos:

$(\mathrm{ECI})=[\mathrm{GP} / \mathrm{Al} / \mathrm{t} \times 100$

Donde: $\mathrm{GP}$ = Peso ganado. $\mathrm{AI}=$ degradación efectiva. $\mathrm{t}$ = días

\subsection{Determinación de la tasa de crecimiento de las larvas de Musca domestica $\mathrm{L}$.}

4.8.1 Evaluación de peso y talla

Llevar a cabo esta evaluación se procedió a pesar (g) y medir la longitud 5 larvas de $M$. domestica $\mathrm{L}$. por bandeja durante el inicio y fin del proceso de bioconversión.

\subsubsection{Determinación de tasas de crecimiento}

Se siguió el diagrama de flujo realizado en el laboratorio de Biología de la Escuela Profesional de Ingeniería Ambiental y Forestal (EPIAF), ver figura 4. De igual manera, través de las fórmulas planteadas por Wootton (1991), se determinaron las tasas de crecimiento absoluta (TAC) y relativa (TRC) para cada dieta o tratamiento y la tasa de crecimiento instantánea conocida también como tasa de especifica de crecimiento (TCE). 


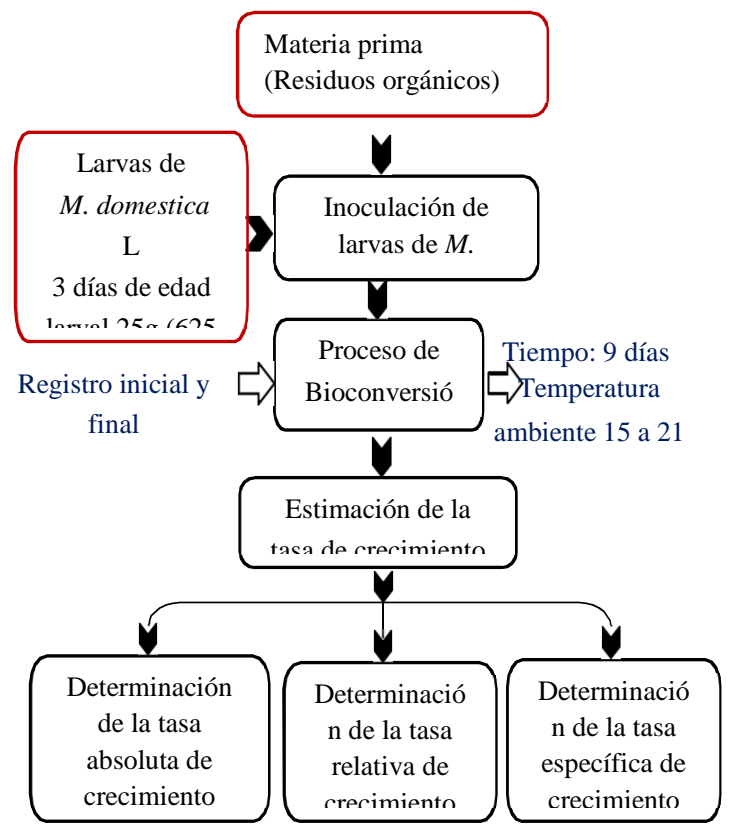

Figura 4. Diagrama de flujo para la determinación de tasas de crecimiento de larvas de $M$. domestica $\mathrm{L}$.

\subsection{Análisis estadístico}

Por medio del Software SPSS versión 25.0 y Minitab versión 18.1.0 se aplicó un Análisis de varianza (ANOVA) con un diseño completo al azar (DCA), con la intención de evidenciar las diferencias significativas entre las medias de los tratamientos. Los análisis se realizaron con un nivel de significancia de 0.05 .

\subsubsection{Modelo estadístico asociado al diseño \\ $Y i j=\mu+\tau i+E i j$ \\ $i=1,2,3, \ldots, t$ \\ $j=1,2,3, \ldots, n$}

Donde: $Y i j=$ Variable respuesta en la j-ésima repetición del iésimo tratamiento. $\mu=$ Media general. $\tau i=$ Efecto del tratamiento i. $E i j=$ Es el error experimental de la unidad ij.

\subsubsection{Transformación angular}

Los datos para el análisis estadístico fueron modificados a una transformación angular o arcoseno, que es empleado en datos aproximados más a una distribución binomial, como el caso de conteos o porcentajes, con el fin de que la distribución de los datos se normalice y estabilizar las varianzas.

\section{Resultados y discusión}

\subsection{Degradación de residuos sólidos}

Los resultados relacionados con la degradación efectiva de residuos por parte de las larvas de mosca doméstica se pueden ver en la tabla 4. Después de nueve (9) días de experimentación, que es el tiempo correspondientes al ciclo larval de la mosca doméstica (M. domestica L.) se inocularon los residuos sólidos estudiados para su degradación con aproximadamente 625 larvas de mosca doméstica, con un peso total de 25g, reportándose que los residuos domiciliarios (RD) fueron los que mayor degradación efectiva presentaron con $107.4 \mathrm{~g}$ que se convirtieron en el mejor tratamiento, ver figura 5 , seguido de los residuos de mercado (RM) con $95.0 \mathrm{~g}$ y por último los residuos de restaurante con $56.2 \mathrm{~g}$. Estos resultados sugieren que las larvas en estudio presentan un nivel de degradación que difiere según el tipo de sustrato. Se reporta que los (RD) presentaron la mayor eficiencia, donde estas larvas llegaron a degradar hasta 4 veces su peso inicial durante el ciclo vital.

Tabla 4. Degradación efectiva y total de residuos sólidos

\begin{tabular}{|c|c|c|c|c|c|c|}
\hline 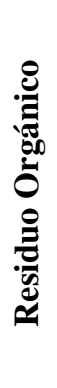 & 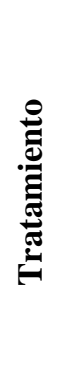 & 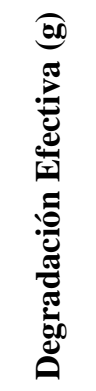 & 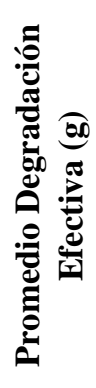 & 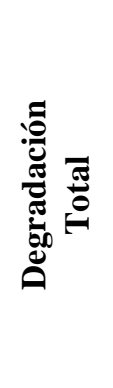 & $\frac{\sqrt[3]{2}}{\frac{2}{2}}$ & 要 \\
\hline \multirow{3}{*}{ 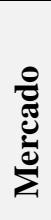 } & $\mathrm{T} 1$ & 97.2 & \multirow{3}{*}{95.0} & 0.0948 & 1.1230 & \multirow{3}{*}{1.1674} \\
\hline & $\mathrm{T} 1$ & 98.7 & & 0.0962 & 1.1664 & \\
\hline & $\mathrm{T} 1$ & 89.1 & & 0.0869 & 1.2130 & \\
\hline \multirow{3}{*}{ 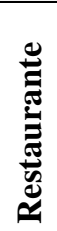 } & $\mathrm{T} 2$ & 56.4 & \multirow{3}{*}{56.2} & 0.0560 & 0.6113 & \multirow{3}{*}{0.6092} \\
\hline & $\mathrm{T} 2$ & 57.4 & & 0.0534 & 0.6222 & \\
\hline & $\mathrm{T} 2$ & 54.8 & & 0.1001 & 0.5940 & \\
\hline \multirow{3}{*}{ 串 } & T3 & 102.6 & \multirow{3}{*}{107.4} & 0.1001 & 1.0536 & \multirow{3}{*}{1.0298} \\
\hline & $\mathrm{T} 3$ & 111.9 & & 0.1091 & 1.0699 & \\
\hline & T3 & 107.6 & & 0.1049 & 0.9658 & \\
\hline
\end{tabular}

Fuente: Elaboración propia. 


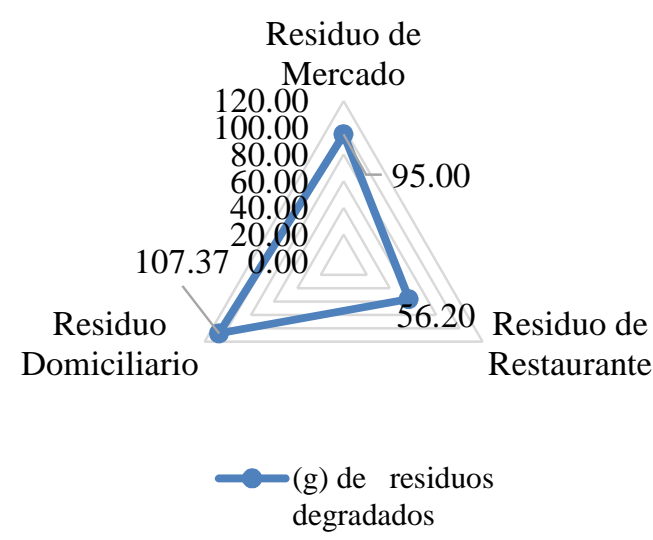

Figura 5. Degradación efectiva de larvas de $M$. domestica $\mathrm{L}$. Fuente: Elaboración propia.

Los resultados de este estudio muestran que los residuos de restaurante fueron los de menor degradación efectiva presente. En Freitas et al. [19], se afirma que el éxito de un organismo o una comunidad biodegradadora va a depender de un conjunto de factores limitantes, entre los que se encuentran las condiciones ambientales, la composición de la materia orgánica, el acceso limitado a los alimentos por el alto contenido de humedad y grasas, el tamaño de las partículas y el contenido de humedad, entre otros parámetros, lo que puede perturbar en el sistema la comunidad de organismos, influyendo en la degradación de residuos. Datos similares fueron reportados en la Meneguz et al. [20], donde se afirma que el tipo de alimento puede influir en el rendimiento de las larvas, reportando que la calidad del sustrato afecta el crecimiento de las moscas soldado negra (MSN). Por ejemplo, si la dieta alimenticia consta de harina de carne, carne de cerdo, pescado o hígado, estas por ser demasiado gruesas, el consumo de estos por parte de las larvas se ve reducido, resultados que están asociados con los resultados reportados en los residuos de restaurante en esta investigación, donde las larvas alimentadas con este tipo de residuo exhibieron el menor índice de reducción de residuos debido a la saturación de harinas y grasas.

\subsection{2 Índice de reducción de residuos sólidos orgánicos}

A través del Análisis de Varianza se pudo demostrar la presencia de diferencias significativas entre los índices de reducción de residuos (WRI). Por lo tanto, se optó por aplicar la prueba de Tukey $(\mathrm{p} \leq 0.05)$ para el índice de reducción de residuos orgánicos, lo que permitió realizar múltiples comparaciones asociadas a las medias de cada uno de los tratamientos, donde se muestra el \% de degradación por día de residuos orgánicos para cada tratamiento, observando un 1.164 \%/día en los residuos domiciliarios (RD), donde este el mejor tratamiento, seguido por el residuos de mercado (RM) con $1.030 \% / d i ́ a, y$ por último los residuos de restaurante (RR) con el menor valor reportado de $0.609 \% /$ día. Estos resultados evidencian que el índice de reducción de residuos orgánicos varía en relación directa al tratamiento, ver figura 6.

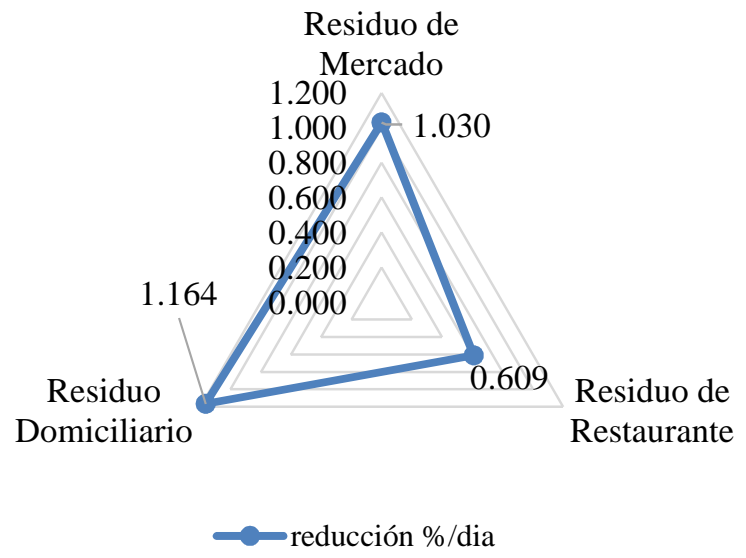

Figura 6. Índice de reducción de residuos sólidos orgánicos.

Fuente: Elaboración propia.

En un estudio realizado por Ground et al. [9] donde se evaluó el índice de reducción de residuos (WRI) de café molido, se reportó valores entre 0.83 y $4 \% /$ día, valores superiores a los reportados en este estudio, probablemente debido a ciertos factores como la presencia de variadas tasas de alimentación, la humedad del material o contenido de fibra, pero sobre todo por la capacidad que presenta cada especie para degradar el residuo orgánico. Esto concuerda con los expuestos en la referencia, que son quienes sostienen que una eficiente transformación de materia orgánica dependerá de la naturaleza física y química del residuo, de los organismos que intervienen durante el proceso de transformación, pero sobre todo de las condiciones físico químicas del proceso.

Por otra parte, Manurung et al. [21] sobre un estudio de bioconversión de residuos orgánicos a través de larvas de mosca soldado negro (Hermetia illucens L.) alimentada con seis cantidades de alimentos $(12,5,25,50,100$, y 200 $\mathrm{mg}$ /larvas/día) hasta que estas alcanzaran el estado de prepupa, se evidenció que la eficiencia de reducción de residuos más alta fue reportada en larvas con una velocidad de alimentación de $12,5 \mathrm{mg} / \mathrm{larva} / \mathrm{día}$ con un índice de reducción de residuos (WRI) de $0.58 \% / d i ́ a$, valor inferior a los alcanzados en esta investigación, donde el residuo de restaurante (RR) fue el que exhibió un índice de reducción menor con un valor de 0.609

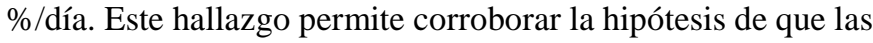
características de la materia orgánica y de los organismos que intervienen, así como de las condiciones físicas químicas del 
proceso como humedad, aireación, temperatura y $\mathrm{pH}$ son condicionantes para una efectiva de residuos orgánicos.

\subsubsection{Eficiencia de conversión de los alimentos ingeridos (ECI)}

Según los resultados obtenidos del análisis de varianza, se reportó que no hay diferencia significativa entre la eficiencia de conversión de alimentos ingeridos (ECI) para los diferentes tratamientos, se optó por aplicar la prueba de Tukey $(\mathrm{p} \leq 0.05)$ para analizar la eficiencia de conversión de alimentos ingeridos por las larvas según el tratamiento, lo que permitió realizar múltiples comparaciones, según las medias de cada tratamiento, ver tabla 6 , donde se observa una eficiencia de conversión en los residuos domiciliarios (RD) de $11.840 \%$, es este el mejor tratamiento en la conversión de alimentos ingeridos por las larvas de mosca doméstica, seguido de un $10.661 \%$ para los residuos de restaurante (RR), y por último un $9.472 \%$ para residuos de mercado (RM), es el que menor eficiencia presentó en la conversión de alimentos ingeridos, ver figura 7.

Tabla 6. Prueba de Tukey ( $\mathrm{p} \leq 0.05)$ eficiencia de conversión de alimentos

\begin{tabular}{|c|c|c|c|c|c|}
\hline & & & ngeridos ( & & \\
\hline 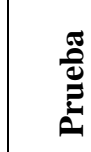 & 苋 & Z & 莺 & $\underbrace{0}_{0=1}$ & 苞 \\
\hline \multirow{3}{*}{$\begin{array}{l}\text { 总 } \\
\text { 至 }\end{array}$} & RD & 3 & 20.060 & 11.840 & A \\
\hline & RR & 3 & 19.057 & 10.661 & A \\
\hline & RM & 3 & 17.922 & 9.472 & A \\
\hline
\end{tabular}

Fuente: Elaboración propia.

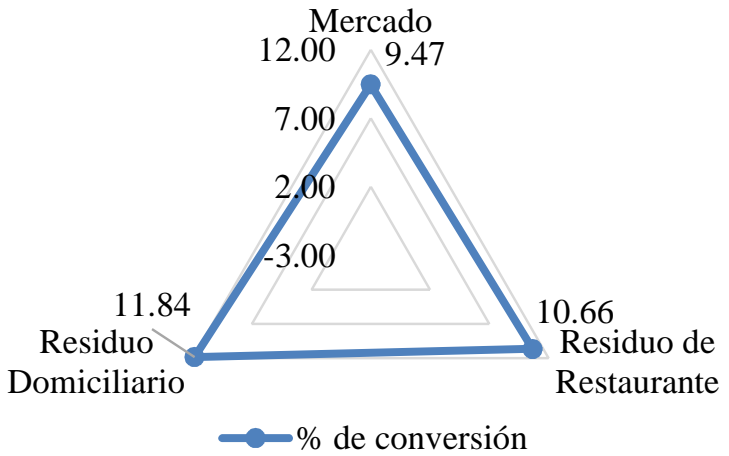

Figura 7. Eficiencia en la conversión de alimentos ingeridos.

Fuente: Elaboración propia.

Durante un estudio de la evaluación de la eficiencia de conversión alimenticia de residuos orgánicos de café molido
[9], obtuvieron una eficiencia de conversión entre $2.71 \%$ - 5\%, resultado diferente al obtenido en el presente estudio, donde las larvas de mosca doméstica presentaron un índice de conversión de alimentos ingeridos superior con valores entre $9.47 \%$. $11.84 \%$, es el residuo de origen domiciliario el de mejor eficiencia, resultado que puede afirmar la anterior explicación relacionada con el balance de masa, aseverando que cuando el alimento se da en pequeñas cantidades se genera una alta cantidad de alimento que es utilizado por el metabolismo.

\subsection{Tasas de crecimiento de larvas de Musca domestica $\mathrm{L}$. \\ 5.2.1 Incremento en longitud de larvas de Musca domestica $\mathrm{L}$.}

Con respecto al incremento de la longitud total, se reportó para los residuos de origen domiciliario (RD) longitudes superiores en larvas alimentadas con este tipo de residuo con un $(\mathrm{p}<0.05)$. De igual manera, se pudo observar diferencias significativas entre las longitudes de las crías y el tipo de tratamiento, ver tabla 7. Se encontraron diferencias significativas con un $\mathrm{p}<0.05$ entre los tratamientos y las tasas de crecimiento absoluta (TAC), relativa (TRC) y específica (TEC), ver tabla 8. Las diferencias encontradas están directamente relacionadas con una mayor cantidad de proteínas en la dieta. Es decir, cuanto mayor es la concentración de proteínas en los alimentos, mayores serán las tasas de crecimiento. Esto es corroborado a través de los resultados, donde se observó un máximo incremento diario de longitud total de $0.333 \mathrm{~mm} /$ día en el tratamiento con residuos domiciliarios (RD), seguido de $0.220 \mathrm{~mm} /$ día para residuos de restaurante (RR) y un mínimo para los residuos de mercado (RM) con un incremento en longitud de $0.160 \mathrm{~mm} /$ día, lo que significa un crecimiento relativo (CR) del $26 \%, 17 \%$ y $12 \%$ respectivamente.

Tabla 7. Tasa de crecimiento absoluta (TAC), relativa (TRC) y específica (TEC) absoluta en longitud total (mm) de larvas de Musca domestica $\mathrm{L}$.

\begin{tabular}{|c|c|c|c|}
\hline 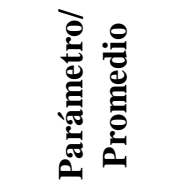 & 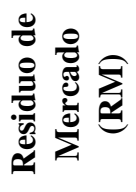 & 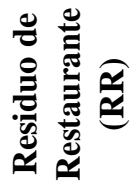 & 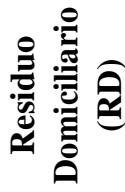 \\
\hline $\begin{array}{c}\text { Talla inicial } \\
\text { promedio } \\
(\mathrm{mm})\end{array}$ & 12.055 & 11.659 & 11.721 \\
\hline $\begin{array}{l}\text { Talla final } \\
\text { promedio } \\
(\mathrm{mm})\end{array}$ & 13.499 & 13.637 & 14.715 \\
\hline $\begin{array}{l}\text { Crecimiento } \\
\text { absoluto } \\
(\mathrm{mm})\end{array}$ & 1.444 & 1.977 & 2.994 \\
\hline
\end{tabular}


Salas (et al): Evaluación del uso de larvas de Mosca doméstica (Musca domestica L.) como tratamiento biológico de residuos orgánicos municipales

\begin{tabular}{|c|c|c|c|}
\hline $\begin{array}{c}\text { Tasa } \\
\text { absoluta de } \\
\text { crecimiento } \\
\text { TAC } \\
\text { (mm/día) }\end{array}$ & 0.160 & 0.220 & 0.333 \\
\hline $\begin{array}{c}\text { Crecimiento } \\
\text { relativo (\%) }\end{array}$ & 12 & 17 & 26 \\
\hline $\begin{array}{c}\text { Tasa relativa } \\
\text { de } \\
\text { crecimiento } \\
\text { TRC (\%) }\end{array}$ & 1.333 & 1.880 & 2.840 \\
\hline $\begin{array}{c}\text { Tasa } \\
\text { específica de } \\
\text { crecimiento } \\
\text { TEC (\%/día) }\end{array}$ & 1.260 & 1.740 & 2.530 \\
\hline $\begin{array}{c}\text { Tiempo } \\
\text { (días) }\end{array}$ & 9 & 9 & 9 \\
\hline
\end{tabular}

Fuente: Elaboración propia.

Según Meneguz et al. [20], en larvas de mosca soldado negro y dietas con altas concentraciones de proteína y grasas, se generan mayores tasas de crecimiento en menor tiempo, lo que refleja posibles diferencias entre los sustratos y en el contenido de nutrientes como carbohidratos estructurales y no estructurales, aminoácidos e inclusive en términos de digestibilidad de los nutrientes. Datos similares se observaron en esta investigación con larvas de mosca doméstica, donde se observó un patrón en el crecimiento larval de rápido aumento en la longitud en larvas obtenidas en sustratos de restaurante, superior a los residuos de mercado. A pesar de tener una rápida tasa de crecimiento, fue el menor peso reportado en contraste con los obtenidos en los demás tratamientos.

Estos resultados son aseverados a través de los resultados obtenidos por Manurung et al [21], donde se evaluó si el tipo de sustrato de cría y su composición de macronutrientes influye en el crecimiento de Hermetia illucens (MSN), estudio que se realizó a través de un índice de crecimiento por tiempo, que midió el crecimiento larvario como el tiempo del desarrollo larvario. Este índice identificó a la "mejor dieta", como aquella en la que las larvas de MSN convierten la mayor biomasa en menor tiempo, el cual fue altamente diferente de manera significativa $(\mathrm{F}(5.30)=19.43 ; \mathrm{p}<0,001)$ entre los grupos experimentales, demostrando que cualquier dieta con grano gastado, ya sea solo o en combinación con fruta, arroja resultados con un valor más alto de este índice, mientras que todas las demás dietas obtuvieron los valores más bajos, lo que refleja que el tipo de sustrato y su composición afectará el tiempo del ciclo vital, lo que se ve reflejado en una mayor talla en menor tiempo.
Tabla 8. Análisis estadístico de las tasas de crecimiento de larvas (Prueba de Tukey) - Tasa de crecimiento absoluta (TAC), relativa (TRC) y específica

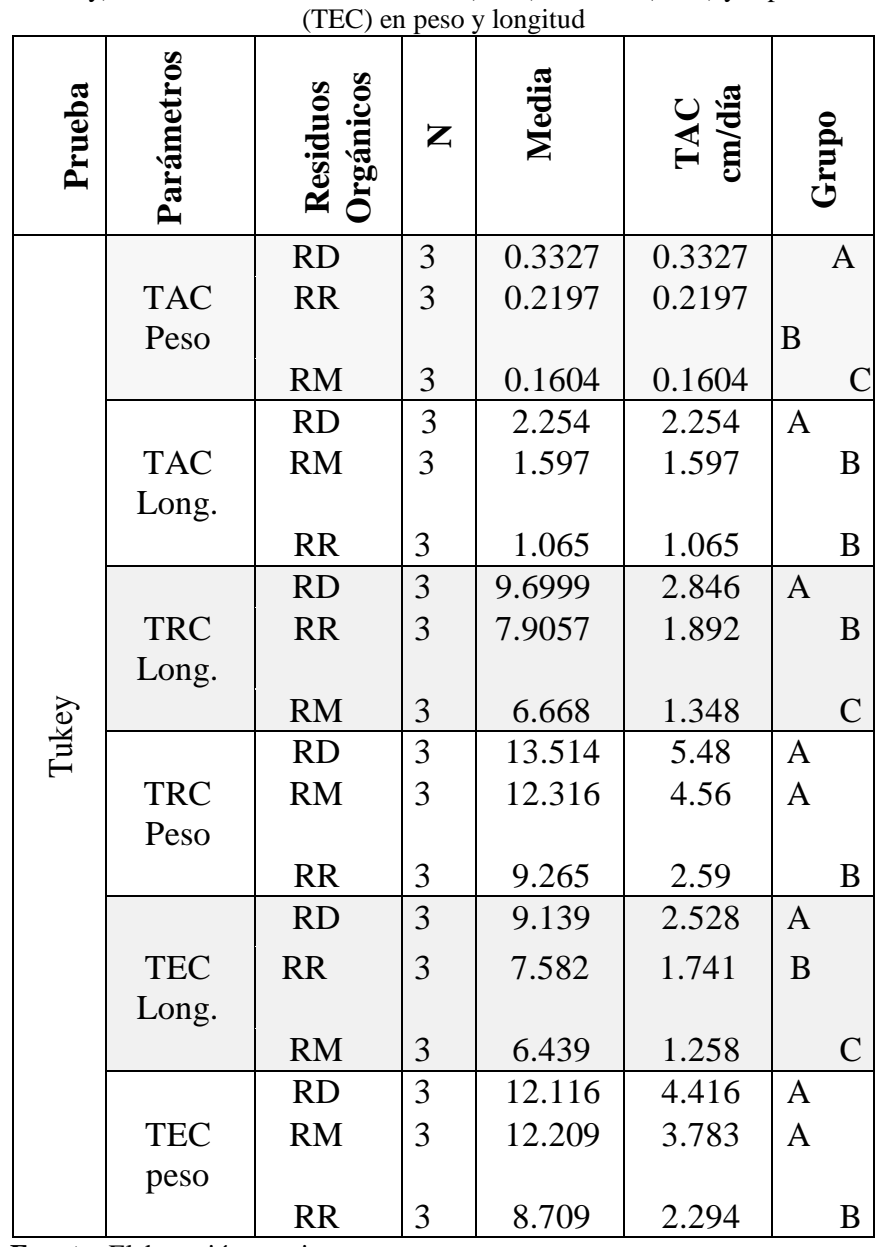

Fuente: Elaboración propia.

\subsubsection{Incremento en peso de larvas de Musca domestica $\mathrm{L}$.}

Los mejores resultados $(\mathrm{p}<0.05)$ se presentaron en larvas alimentadas con residuos domiciliarios, los cuales presentan un mayor contenido de proteínas y de lípidos, así como una menor cantidad de carbohidratos. Asimismo, no se encontraron diferencias estadísticas significativas $(p>0.05)$ entre las tasas relativas de crecimiento (TAC) y tasas específicas de crecimiento entre larvas alimentadas con residuos domiciliarios y de mercado. El incremento diario máximo en peso fue de $2.254 \mathrm{mg} / \mathrm{día}$ con residuos domiciliarios (RD), seguido de residuos de mercado (RM) con 1.597 y el menor $1.065 \mathrm{mg} / \mathrm{día}$ con residuos orgánicos de restaurante (RR), lo anterior representa una diferencia de $27.50 \%$ a favor de los primeros $(\mathrm{p}<0.05)$. Esto representa un crecimiento relativo (CR) del $49.6 \%, 40.382 \%$ y $23.3 \%$ respectivamente, ver tabla 9. 
Salas (et al): Evaluación del uso de larvas de Mosca doméstica (Musca domestica L.) como tratamiento biológico de residuos orgánicos municipales

Tabla 9. Tasa de crecimiento absoluta (TAC), relativa (TRC) y específica (TEC) en peso (mg) de larvas de Musca domestica $\mathrm{L}$ :

\begin{tabular}{|c|c|c|c|}
\hline 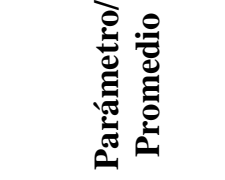 & 刍 & 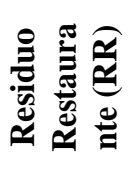 & 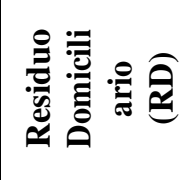 \\
\hline $\begin{array}{c}\text { Peso inicial } \\
(\mathrm{mg})\end{array}$ & 35.593 & $\begin{array}{c}41.0 \\
93\end{array}$ & 40.907 \\
\hline Peso final (mg) & 49.967 & $\begin{array}{c}50.6 \\
81\end{array}$ & 61.193 \\
\hline $\begin{array}{c}\text { Ganancia en } \\
\text { peso }(\mathrm{mg})\end{array}$ & 14.373 & $\begin{array}{c}9.58 \\
8 \\
\end{array}$ & 20.287 \\
\hline $\begin{array}{l}\text { Tasa absoluta } \\
\text { de crecimiento } \\
\text { TAC (mg/día) }\end{array}$ & 1.597 & $\begin{array}{c}1.06 \\
5\end{array}$ & 2.254 \\
\hline $\begin{array}{l}\text { Crecimiento } \\
\text { relativo }(\%)\end{array}$ & 40.382 & $\begin{array}{c}23.3 \\
32 \\
\end{array}$ & 49.593 \\
\hline $\begin{array}{c}\text { Tasa de } \\
\text { crecimiento } \\
\text { relativo TCR } \\
(\%)\end{array}$ & 4.487 & $\begin{array}{c}2.59 \\
2\end{array}$ & 5.510 \\
\hline $\begin{array}{c}\text { Tasa especifica } \\
\text { de crecimiento } \\
\text { TEC (\%/día) }\end{array}$ & 3.783 & $\begin{array}{c}2.29 \\
4\end{array}$ & 4.416 \\
\hline Tiempo (días) & 9 & 9 & 9 \\
\hline
\end{tabular}

Fuente: Elaboración propia.

El peso y el rendimiento de las larvas fueron afectados por la concentración de nutrientes en las tres densidades de larvas, en donde las larvas obtenidas en el sustrato de restaurante que presentaron un menor aumento de peso debido a la sobresaturación de grasas. Según Manurung et al. [21] larvas alimentadas con $200 \mathrm{mg}$ de paja de arroz molida al día presentan un peso seco prepupal más alto en un tiempo de desarrollo más corto, con una media de $13.64 \mathrm{mg}$ y 38 días, respectivamente, lo que demuestra que la disminución de la cantidad de alimento produce un menor peso prepupal y un mayor tiempo de desarrollo.

Según Beniers \& Graham [22] en el estudio sobre el efecto de diferentes dietas con una variedad de concentraciones de proteínas y carbohidratos y su efecto en el crecimiento y composición de las larvas de la mosca soldado negra $(H$. illucens), se demostró que tanto la proteína de la dieta como los carbohidratos de la dieta, afectan significativamente el peso fresco y seco de las larvas, en donde es la proteína de la dieta el indicador más fuerte del peso fresco y seco de las larvas que los carbohidratos de la dieta, por lo que la composición de las larvas se vio influenciada por el tipo de alimento, obteniendo larvas más pesadas, lo que significa más grasa que las de menor peso, mientras que la concentración de cenizas fue muy estable a lo largo de su ciclo de vida.

\subsection{Mortalidad y supervivencia de larvas de Musca domestica $\mathbf{L}$.}

Con respecto a los resultados sobre mortalidad, supervivencia y transformación de las larvas de mosca doméstica durante su fase de crecimiento, se pudo observar una variación entre los diferentes tipos de residuos, ver tabla 10. En lo que corresponde a los resultados alcanzados por larvas alimentadas con residuos domiciliarios (RD) y residuos de restaurante (RR) estas no mostraron mortalidad alguna, en ambos tratamientos son eficaces para el crecimiento de larvas de $M$. domestica L., y los residuos de mercado (RM) el último tratamiento con un $80 \%$ de supervivencia y $20 \%$ de mortalidad, en el cual se evidenció la muerte de larvas durante los primeros días de inoculación, hecho que sea posiblemente consecuencia del proceso de selección realizada en la fase de aclimatación y de la tolerancia de los alimentos y de las características físicas y químicas del residuo orgánico [22].

En estudio con tratamientos de diferentes $\mathrm{pH}$ se demostró como este factor influye en el peso de las larvas el primer, tercer y quinto día, pero no al final del ensayo, en donde se observa que la actividad larvaria aumentó los valores de $\mathrm{pH}$ a partir del cuarto día, con valores finales de alrededor de $8,9 \pm 9,4$ en todos los tratamientos, además de una variación del peso de las prepupas entre 0.094 y 0.100 g. Se observó, que el peso final de las larvas y pupas, la proporción sexual, la cantidad de alimento ingerido, la mortalidad de las larvas, y el tiempo que necesitaron para alcanzar el estadio de pupa se vieron afectados por el tipo de alimento, lo que demuestra evaluar adecuadamente el tipo de alimento con el que se alimentarán las larvas y el valor inicial del $\mathrm{pH}$ del sustrato son parámetros importantes para reducir el tiempo y aumentar el peso en la producción de larvas [23].

Tabla 10. Porcentaje de mortalidad y supervivencia de larvas de Musca

\begin{tabular}{|c|c|c|c|c|c|c|}
\hline 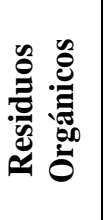 & 苍 & o & o & o & 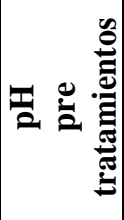 & 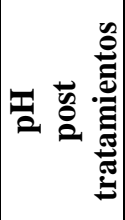 \\
\hline \multirow{3}{*}{ 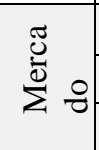 } & $\mathrm{T} 1$ & 25.60 & 74.40 & 3.2 & 5.4 & 6.1 \\
\hline & $\mathrm{T} 1$ & 25.28 & 74.72 & 4.0 & 5.2 & 6.0 \\
\hline & T1 & 27.52 & 72.48 & 3.2 & 5.3 & 6.0 \\
\hline \multirow{3}{*}{ 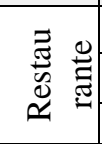 } & $\mathrm{T} 2$ & 0.0 & 100.0 & 0.0 & 6.0 & 5.2 \\
\hline & $\mathrm{T} 2$ & 0.0 & 100.0 & 0.0 & 6.2 & 5.1 \\
\hline & $\mathrm{T} 2$ & 0.0 & 100.0 & 0.0 & 6.1 & 5.0 \\
\hline \multirow{3}{*}{ 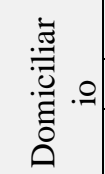 } & T3 & 0.0 & 100.0 & 16.0 & 6.3 & 7.2 \\
\hline & T3 & 0.0 & 100.0 & 16.8 & 6.1 & 7.1 \\
\hline & T3 & 0.0 & 100.0 & 15.2 & 6.0 & 7.0 \\
\hline
\end{tabular}

Fuente: Elaboración propia. 
Según datos reportados en Arroyave Sierra et al. [24], durante un estudio sobre el crecimiento de larvas de mosca soldado con diferentes alimentos y durante cuatro semanas, se observó que la alimentación con porcinaza produce larvas con menor desempeño en peso, largo y ancho, comparado con las alimentadas con gallinaza y alimento para ponedoras, los cuales no presentan diferencias significativas, lo que indica que la gallinaza y el alimento para ponedoras son más eficientes en términos de crecimiento, por su composición presentan un excelente valor nutricional y de elevada digestibilidad, con un adecuado contenido de proteína y carbohidratos para la alimentación de las larvas y en el caso de la gallinaza por estar enriquecida microbiológicamente, lo que contribuye con las concentraciones de nutrientes de para ser consumidas por las larvas.

Con respecto a la humedad como factor limitante para la supervivencia de larvas de insectos, según Tadayyon et al. [15] se demostró que este es otro factor de gran importancia para la sobrevivencia de las larvas. Si esta es baja, estas larvas pueden secarse, mientras que un medio de crecimiento con demasiada humedad conduce a su ahogamiento. En relación con la transformación de larvas de mosca doméstica a pupa durante un tiempo de nueve (9) días, periodo que duró la experimentación, la mayor transformación en pupas se comprobó en residuos domiciliarios (RD) con $16 \%$ seguido de residuos de mercado (RM) con $3.3 \%$ y por último residuos de restaurante (RR), en donde las pupas estuvieron ausentes. Debido a que los residuos presentaban mayor humedad, quienes sostienen que las pupas soportan una menor humedad para su desarrollo, donde esta humedad esencial en el proceso de metamorfosis en pupas de larvas, por lo que este factor está relacionado con la duración de los estados de larva y pupa de las moscas domésticas.

Por su parte, en Gold et al. [25] evaluaron ciertos parámetros de rendimiento en larvas de soldado negro, tales como: la supervivencia y la tasa de bioconversión, la reducción de residuos y la eficiencia de conversión de residuos y proteínas, alimentadas con diferentes fórmulas alimenticias, reportando que en comparación con los piensos usados para alimentación de aves de corral, los residuos de origen vegetal son los que generan mejores resultados, con el peor obtenido del estiércol de vaca. Esto demuestra un mejor rendimiento y una menor variabilidad en comparación con los residuos individuales, que es una de las razones de esta variabilidad del contenido en fibra y lípidos, y así generar una orientación sobre cómo el funcionamiento sistemático de los tratamientos más adecuados a utilizar basados en el uso de biorresiduos de diferente composición y origen.

\section{Conclusiones}

- Las larvas de la mosca doméstica (M. domestica L.), son capaces de digerir y degradar residuos orgánicos de manera eficaz. El índice de reducción de residuos orgánicos varía en relación directa al tipo de residuo empleado y los residuos domiciliarios (RD) fueron el mejor tratamiento, con un índice de reducción de 1.167 \%/día, donde las larvas degradan hasta 4 veces su peso en un periodo de vida de 9 días.

- En relación con la conversión de alimentos ingeridos por larvas de mosca doméstica, se demostró estadísticamente que no existe diferencia significativa entre los tratamientos, sin embargo, el mejor tratamiento fue el de residuos domiciliarios (RD) con $11.840 \%$.

- En relación con el incremento de longitud de larvas de mosca doméstica, las tasas de crecimiento absoluta (TAC), relativa (TRC) y específica (TEC) fueron significativamente diferentes $(\mathrm{p}<0.05)$ en todos los tratamientos. El tipo de sustrato afectó significativamente la capacidad de las larvas para desarrollarse con respecto a la tasa de crecimiento, la mortalidad, y la transformación en pupas.

- Cuanto mayor es la tasa de crecimiento de las larvas de Mosca doméstica, más alto es el índice de reducción de residuos orgánicos.

\section{AGRADECIMIENTOS}

A la Universidad Nacional de Juliaca, Perú por las herramientas por el apoyo institucional y durante la formación profesional.

A los docentes de la Escuela Profesional Ingeniería Ambiental y Forestal, por los conocimientos impartidos que permitieron cumplir con esta investigación.

\section{REFERENCIAS}

[1] M. A. Vitorino de Souza, S. Montenegro, K. Faceli \& V. Casadei. Technologies and decision support systems to aid solid-waste management: a systematic review. Vol. 59, pp. 567584. 2017. [Online]. Available: Doi: https://doi.org/10.1016/j.wasman.2016.10.045.

[2] M. Neugebauer \& P. Sołowiej. El uso de residuos verdes para superar la dificultad del compostaje a pequeña escala de residuos orgánicos domésticos. Journal of Cleaner Production, no.156, pp. 865-875. 2017. [Online]. Available: doi: 10.1016/ j.jclepro.2017.04.095.

[3] E. Kazuva \& J. Zhang. Analyzing Municipal Solid Waste Treatment Scenarios in Rapidly Urbanizing Cities in Developing Countries: The Case of Dar es Salaam, Tanzania. International Journal of Environmental Research and Public Health, vol.16, no. 11, pp.20-35. 2019. [Online]. Available: doi:10.3390/ijerph16112035.

[4] P. S. Michel Devadoss, P. Agamuthu, S. B. Mehran, C. Santha \& S. H. Fauzia. Implications of municipal solid waste management on greenhouse gas emissions in Malaysia and the 
way forward. Waste Management, vol. 119, pp.135-144. 2021. [Online]. Available: doi: 10.1016/ j.wasman.2020.09.038.

[5] R. Salomone, G. Saija, G. Mondello, A. Giannetto, S. Fasulo \& D. Savastano. Environmental impact of food waste bioconversion by insects: Application of Life Cycle Assessment to process using Hermetia illucens. Journal of Cleaner Production, vol. 140, pp. 890-905. 2021. [Online]. Available: doi: 10.1016 / j.jclepro.2016.06.154.

[6] N. Fadhillah \& A. Y. Bagastyo. Utilization of Hermetia illucens Larvae as A Bioconversion Agent to Reduce Organic Waste IOP Conference Series: Earth and Environmental Science, vol. 506, 012005. 2020. [Online]. Available: doi: 10.1088 / 1755$1315 / 506 / 1 / 012005$.

[7] A. Van Huis \& D. G. Oonincx. The environmental sustainability of insects as food and feed. A review. Agronomy for Sustainable Development, vol.37, no. 5, pp. 43 .2017.

[8] A. Kahar, M. Busyairi, S., Agus Hermanto, A. Ristanti. Bioconversion of municipal organic waste using black soldier fly larvae into compost and liquid organic fertilizer. Konversi, vol. 9, no. 2, pp. 35-40. 2020. [Online]. Available: Doi: 10.20527/k.v9i2.9176.

[9] C. Ground, A. D. Permana, J. Esther \& N. R. Eka. Growth of Black Soldier Fly (Hermetia illucens) Larvae Fed on Spent Growth of Black Soldier Fly (Hermetia illucens) Larvae Fed on Spent Coffee Ground. 2018. [Online]. Available: https://doi.org/https://doi.org/10.1088/17551315/187/1/012070

[10] Y. Niu, D. Zheng, B. Yao, Z. Cai, Z. Zhao, S. Wu, ... D. Yang. A novel bioconversion for value-added products from food waste using Musca domestica. Waste Management, vol.61, pp. 455-460. 2017. [Online]. Available: doi:10.1016/j.wasman.2016.10.054)

[11] M. Limas \& J. E. Blanco. Prácticas de consumo-desecho de residuos sólidos domiciliarios en Ciudad Juárez en 2014. Iztapalapa. Revista de ciencias sociales y humanidades, vol.38, no.83, pp. 97-132. 2017. [Online]. Available: https://dx.doi.org/10.28928/revistaiztapalapa/832017/atc4/blan coromeroje/limashernand ezm.

[12] J. Vázquez, M. Álvarez-Vera, S. Iglesias-Abad \& J. Castillo. La incorporación de enmiendas orgánicas en forma de compost y vermicompost reduce los efectos negativos del monocultivo en suelos. Scientia Agropecuaria, vol.11, no.1, pp. 105-112. 2020. [Online]. Available:

Doi https://doi.org/10.17268/sci.agropecu.2020.01.12.

[13] K. Paritosh, M. Yadav, S. Mathur, V. Balan \& W. Liao. Organic Fraction of Municipal Solid Waste: Overview of Treatment Methodologies to Enhance Anaerobic Biodegradability, vol.6, pp. 1-17. 2018. [Online]. Available: Doi https://doi.org/10.3389/fenrg.2018.00075.

[14] Y. S. Wang \& M. Shelomi. Review of Black Soldier Fly (Hermetia illucens) as Animal Feed and Human Food. Foods, vol.6, no.10, pp91. 2017. [Online]. Available: 10.3390/foods6100091. PMID: 29057841; PMCID: PMC5664030.

[15] Tadayyon, A., Naeimi, M. M., \& Pessarakli, M. Effects of vermicompost and vermiwash biofertilizers on fenugreek (Trigonella foenum) plant. Communications in Soil Science and Plant Analysis, vol. 49, no. 19, pp. 2396-2405. 2018. [Online]. Available: https://doi.org/10.1080/00103624.2018.1510950.

[16] A. Meraz, A. López, C. A. García, J. A, Torres \& M. A. García. Distribución potencial de Musca doméstica en el municipio de
Jesús María, Aguascalientes, con el uso de escenarios de cambio climático. Revista Mexicana de Ciencias Pecuarias, pp.14-29. 2019. [Online] Available Doi: https://doi.org/http://dx.doi.org/10.22319/rmcp.v10i1.4241.

[17] H. Villegas. Mosca Domestica Biología y Control. Facultad de Ciencias Biológicas. Universidad Autónoma de Nuevo León. C, vol. 8, no. 2, pp.11-29. 2017. [Online]. Available: http://artropodosysalud.com/Publicaciones/No8Dic2017/4Mosca_Domestica.pdf.

[18] R. Hernández, C. Fernández \& P. Baptista. Metodología de la investigación. Journal of Chemical Information and Modeling . Vol. 53. 2014. [Online]. Available: Doi: https://doi.org/10.1017/CBO9781107415324.004.

[19] A. F. Freitas, V. D. Leite, M. M. P. da Silva, R. B. Gomes, M. J. Silva, F. L. B. Farias \& A. T. Barros. Aerobic treatment of organic solid waste generated in a vertical condominium as a sustainable alternative. Research, Society and Development, [S. 1.], v. 9, n. 10, p. e8469109168, 2020. [Online]. Available: Doi: 10.33448/rsd-v9i10.9168.

[20] M. Meneguz, A. Schiavone, F. Gai, A. Dama, C. Lussiana M. Renna \& L. Gasco. Efecto del sustrato de cría sobre el rendimiento del crecimiento, la eficiencia de reducción de desechos y la composición química de las larvas de la mosca soldado negra (Hermetia illucens). Revista de Ciencias de la Alimentación y la Agricultura, 2018. [Online] Available: doi: 10.1002 / jsfa.9127.

[21] R. Manurung, A. Supriatna, R. R. Esyanthi \& R. E. Putra. Optimal feed rate for biomass production. 1036 Journal of Entomology and Zoology Studies, vol.4, no. 4, pp. 1036- 1041. 2016. [Online]. Available: http://www.entomoljournal.com/archives/2016/vol4issue4/Part K/4- 3-163-796.pdf

[22] J.J.A. Beniers \& R.I. Graham. Effect of protein and carbohydrate feed concentrations on the growth and composition of black soldier fly (Hermetia illucens) larvae. Journal of Insects as Food and Feed, vol. 5. no. 3, pp 193 - 199. $2019 . \quad$ [Online]. Available: https://doi.org/10.3920/JIFF2018.0001

[23] Meneguz, L. Gasco \& J. K. Tomberlin. Impact of $\mathrm{pH}$ and feeding system on black soldier fly (Hermetia illucens, L; Diptera: Stratiomyidae) larval development. PLoS ONE, vol. 13, no.8. 2018. [Online]. Available: https://doi.org/10.1371/journal.pone.0202591.

[24] O. Arroyave Sierra, J. Chamorro Rengifo \& A. Ochoa Muñoz. Crecimiento de larvas de mosca soldado alimentadas con gallinaza, porcinaza y alimento para ponedoras. Revista Colombiana De Ciencia Animal - RECIA, vol. 11, no.2, pp. 730. $2019 . \quad$ [Online]. Available: https://doi.org/10.24188/recia.v11.n2.2019.730.

[25] M. Gold, C. M. Cassar, C. Zurbrügg, M. Kreuzer, S. Boulos, S. Diener \& A. Mathys. Biowaste treatment with black soldier fly larvae: Increasing performance through the formulation of biowastes based on protein and carbohydrates. Waste Management, vol. 102, pp. 319-329. 2019. [Online]. Available: doi:10.1016/j.wasman.2019.10.036. 